\section{Evolución de un linfoma no Hodgkin "triple expresor" en un paciente trasplantado renal con quimioterapia DA-R-EPOCH. Caso clínico}

\author{
NATALIA ARÁNGUIZ1,2, JORGE VEGA ${ }^{3,4}$
}

\section{Triple expressor lymphoma in a kidney transplant patient}

Patients transplanted from solid organs have an increased risk of cancer, especially lymphomas. Lymphomas correspond to 4 to 5\% of malignant neoplasms in the general population and in solid organ transplant patients it reaches an incidence of $21 \%$. The incidence of non-Hodgkin lymphomas is 10 times higher than in the non-transplanted population. We report the case of a 68-year-old man with a kidney transplant who 6 years after transplantation, developed a non-Hodgkin diffuse large cells B lymphoma with lymph node and pulmonary involvement, with markers of very poor prognosis (triple MYC expressor, BCL2 and BCL6). and its evolution with chemotherapy with DA R EPOCH.

(Rev Med Chile 2019; 147: 247-250)

Key words: Drug Therapy; Kidney Transplantation; Lymphoma, NonHodgkin; Lymphoproliferative Disorders).
'Servicio de Medicina, Sección Hematología, Hospital Dr. Gustavo Fricke, Viña del Mar,

Chile.

2Servicio de Hemato-Oncología, Hospital Naval A. Nef, Viña del Mar, Chile.

${ }^{3}$ Servicio de Medicina, Sección Nefrología, Hospital Naval A. Nef, Viña del Mar, Chile.

${ }^{4}$ Departamento de Medicina, Escuela de Medicina, Universidad de Valparaíso, Chile.

Trabajo no recibió financiamiento. Los autores declaran no tener conflictos de interés.

Recibido el 13 de junio de 2018, aceptado el 4 de enero de 2019.

Correspondencia a:

Jorge Vega

5 Norte 1035. Viña del Mar,

Chile.

jvegastieb@gmail.com
L os pacientes trasplantados de órganos sólidos (TOS) tienen mayor riesgo de sufrir cáncer respecto a la población general, particularmente linfomas ${ }^{1}$. En sujetos inmunocompetentes con tumores malignos, los linfomas corresponden a 4-5\% de los cánceres, en TOS esta proporción aumenta hasta $21 \%^{2}$. La incidencia de linfoma no Hodgkin (LNH) es 10 veces mayor que en la población adulta no trasplantada, la de linfoma de Hodgkin (LH) es 4 veces mayor ${ }^{3}$. En niños trasplantados este riesgo es hasta 120 veces mayor ${ }^{1}$.

Los receptores de injertos renales tienen una incidencia de enfermedad linfoproliferativa post trasplante (PTLD; en la sigla en inglés) de 0,8$2,5 \%$, menor a la de receptores órganos como pulmón, intestino o multiorgánicos que tienen una mayor incidencia ${ }^{4}$.

La infección por el virus Epstein Barr (EBV) se ha asociado al 50\% de PTLD. Los pacientes seronegativos para EBV que reciben un órgano de un donante seropositivo tienen riesgo 10 a 75 veces mayor de PTLD respecto a receptores seropositivos. Los niños (generalmente seronegativos) tienen más frecuentemente PTLD ${ }^{1}$. La inmunosupresión reduce la capacidad de los linfocitos $\mathrm{T}$ para destruir a las células B infectadas por EBV, estas células proliferan y originan linfomas ${ }^{1}$. La patogenia en pacientes seronegativos para EBV es menos clara. Se ha planteado que este virus pudiera iniciar la enfermedad y desaparecer o que participen otros virus (CMV, virus C) o que la causa sea la estimulación inmunológica persistente producida por el injerto ${ }^{6,7}$.

El aumento en la incidencia de PTLD también se ha relacionado con mayor edad de donantes y receptores, aumento en el número de trasplantes, aumento en la sospecha, mejoría en métodos diagnósticos, disfunción inmune secundaria a inmunosupresión crónica, estimulación antigénica crónica por el órgano trasplantado y esquemas inmunosupresores más potentes ${ }^{1,5}$. En receptores añosos se ha invocado la senescencia inmune ${ }^{5}$. 
La inducción con anticuerpos contra linfocitos $\mathrm{T}$ (monoclonales, policlonales), como el uso de azatioprina y tacrolimus, se ha considerado que influyen en el desarrollo de PTLD. Es controversial si la ciclosporina y los inhibidores $\mathrm{mTOR}$ (sirolimus, everolimus) influyen. El micofenolato, basiliximab y daclizumab se considera que no tienen este riesgo ${ }^{1}$.

Se comunica el caso de un paciente trasplantado renal que desarrolló un LNH de alta malignidad, difuso de células grandes B con marcadores inmunohistoquímicos de lo que se ha llamado "triple-expresor", situación que es poco frecuente, de mal pronóstico y que, sin embargo, tuvo una evolución inesperada con un tratamiento innovador.

\section{Caso clínico}

Hombre que ingresó a diálisis peritoneal por una falla renal secundaria a glomerulonefritis crónica permaneciendo en ella 6 años. Recibía fenitoína por epilepsia. Recibió un riñón de donante fallecido a los 62 años. Tenía anticuerpos IgG para EBV y CMV y serología negativa para virus C, B e HIV. Se indujo con basiliximab y utilizó azatioprina, ciclosporina y prednisona. Durante los 5 primeros años su evolución fue normal, salvo una reparación de la anastomosis uretero-vesical y su creatinina se mantenía en 1,4 mg/dl.

$\mathrm{Al}$ sexto año consultó por tos seca, disnea de esfuerzos y baja de peso de 10 kilos en tres meses en ausencia de fiebre, sudoración nocturna y prurito. Existían adenopatías supraclaviculares e inguinales bilaterales de consistencia gomosa. Un TAC mostró adenopatías cervicales, hiliares, mediastínicas, retroperitoneales e inguinales. Se objetivó compromiso pulmonar izquierdo secundario al tumor, el bronquio superior izquierdo estaba comprimido, había derrame pleural ipsilateral de moderada cuantía y el injerto presentaba hidronefrosis. El paciente no presentaba compromiso de sistema nervioso central en el TAC. La biopsia excisional del ganglio inguinal mostró extensa infiltración linfoide atípica de células grandes con escaso citoplasma, núcleos ovalados de cromatina grumosa, nucléolos prominentes, alta actividad mitótica, pero sin necrosis. La inmunohistoquímica mostró CD20 (+), CD5 (-), CD30 (-) y CD10 (+) en más del 80\% de las células neoplásicas. BCL6 fue $(+)$ en más de $80 \%$ de las células (patrón nuclear), BCL2 (+) en más de $50 \%$ (patrón de membrana), factor de proliferación ki67 $(+)$ en más de $90 \%$ (patrón nuclear), C-MYC (+) en más de $80 \%$ (patrón citoplasmático) y EBER (-). La conclusión fue LNH difuso de células grandes $\mathrm{B}$ (DCGB) de alto grado, "triple expresor" (BCL2, C-MYC, BCL6). No se realizó biología molecular por no contar con ella en nuestro centro y no estar incluida en la canasta GES. La biopsia de médula ósea fue normal. Fue clasificado como estadio IVB. El índice pronóstico internacional (score IPI) fue 5 (riesgo elevado) con estimación de sobrevida a 5 años de $26 \%{ }^{8}$.

A su ingreso requirió oxígeno y hemodiálisis (creatinina 5,4 mg/dl). Días después se efectuó un neoimplante ureterovesical y la función mejoró paulatinamente hasta obtener una creatinina 1,5 $\mathrm{mg} / \mathrm{dl}$. La azatioprina se suspendió, manteniendo la prednisona y ciclosporina (en dosis reducidas). Posteriormente, la ciclosporina se cambió por everolimus. En los 6 meses siguientes se administraron 6 ciclos del esquema DA-R-EPOCH (rituximab, ciclofosfamida, vincristina, etoposido, doxorubicina). Durante ellos presentó neutropenias febriles, infecciones urinarias y diarreas por clostridium difficile, que respondieron a los tratamientos. Finalizada la quimioterapia, se efectuó un PET Scan que mostró una adenopatía cervical y supraclaviculares bilaterales con tenue captación de 5 fluoroglucosa (no palpables ni ecográficamente visibles) con un SUV máximo de 3,4 , informándose un score Deauville de 2.

Ocho años después del trasplante y a 25 meses de finalizada la quimioterapia, el paciente se encuentra en buenas condiciones generales, sin evidencia de recidiva de linfoma y con buena función del injerto (creatinina: $1,5 \mathrm{mg} / \mathrm{dl}$ ).

\section{Discusión}

Este paciente, 6 años después del TxR, presentó un LNH de alto grado de malignidad con marcadores de muy mal pronóstico como es la expresión de MYC, BCL2 y BCL6 ("triple expresor") .

En una serie de 178.785 trasplantes de órganos sólidos encontraron 1.617 LNH y $48 \mathrm{LH}$. De los LNH 85,5\% fueron de estirpe B y 6,2\% T. El más frecuente fue DCGB (2/3 del total), como este paciente $^{5}$. La OMS, en su última clasificación 
de PTLD, distinguió 4 subclases. Las 3 primeras asociadas $>90 \%$ a infección por EBV y la restante (como este paciente) en $50 \%{ }^{10}$.

Los pacientes con PTLD tienen una carga viral EBV más alta que los que no tienen la enfermedad ${ }^{1}$.

Los LNH tienen una incidencia con un patrón en " $U$ ", alto riesgo el primer año, luego esta cae, para elevarse 5 a 10 años después ${ }^{5}$. Los pacientes seropositivos para EBV suelen presentar la enfermedad durante el primer año, los seronegativos más frecuentemente entre los 5 y 10 años ${ }^{5}$. Las formas tempranas frecuentemente comprometen al órgano trasplantado e infrecuentemente órganos extranodales ${ }^{1,5}$. Las formas tardías suelen comprometer el tracto gastrointestinal y el sistema nervioso central ${ }^{1}$.

A pesar de los avances en el pronóstico de los LNH desde la introducción de los anticuerpos monoclonales anti-CD20 (rituximab) y su adición al esquema clásico (CHOP), los linfomas con aberraciones MYC, especialmente aquellos con concomitancia de alteraciones de BCL2 y/o BCL6, se asocian a un peor pronóstico ${ }^{11}$. MYC es un factor de transcripción localizado en el cromosoma 8 que regula la expresión de genes involucrados en el ciclo celular, reparación de DNA, síntesis proteica y reacción al estrés. BCL2 tiene funciones antiapoptóticas. BCL6 al sobreexpresarse en las células B evita la apoptosis frente al daño del DNA ${ }^{12}$.

La clasificación de la OMS (revisión 2016) incluyó una nueva categoría de LNH de alto grado con reordenamientos de MYC, BCL2 y BCL6, denominándolos "doble y triple Hit". Es importante diferenciarlos de los linfomas "doble o triple expresores" (como este caso) en los cuales en la inmunohistoquímica hay una expresión aumentada de estos factores en ausencia de su detección por biología molecular o $\mathrm{FISH}^{13}$. En este caso no contamos en nuestro centro con la posibilidad de efectuar dichas detecciones, si bien sí esta disponible en algunos centros públicos con recursos propios ${ }^{14}$. Los LNH "doble o triple expresores" no constituyen una entidad distinta en la última clasificación de la OMS, pero sirven como marcadores de peor pronóstico ${ }^{15}$. A la fecha, no existen comunicaciones de ensayos prospectivos que orienten a la mejor forma de tratar estos linfomas y solo hay reportes de casos, en los cuales se han observado mejores respuestas con esquemas agresivos como el R DA EPOCH ${ }^{16}$. Dado que, a diferencia de LNH doble o triple hit, los escasos reportes de LNH “doble o triple expresores" tratados con R DA EPOCH no incluían profilaxis en sistema nervioso central, esta no se realizó en el paciente ${ }^{17}$. La terapia usual en PTLD es la reducción de la inmunosupresión y la administración de rituximab. Cuando no hay respuesta a este tratamiento se ha recomendado agregar $\mathrm{CHOP}^{1,18}$. La información sobre $\mathrm{LNH}$ "doble o triple expresores" en pacientes trasplantados es muy escasa. Recientemente se comunicó una serie con 6 pacientes trasplantados renales ( 5 tenían un linfoma $\mathrm{B}$ difuso de células grandes) que fueron tratados con reducción de la inmunosupresión y el esquema R DA EPOCH de entrada. Los resultados fueron superiores a los de las series históricas con rituximab o R-CHOP, obteniéndose $70 \%$ de respuestas completas. Ninguno de los casos tenía criterios de mal pronóstico como ser "doble o triple expresor" 19 como este enfermo.

En este paciente, que tenía un mal pronóstico, basándonos en los pocos datos comunicados en la literatura existente acerca de los LNH "doble y triple hit/ doble y triple expresores ${ }^{16}$, se decidió tratarlo con quimioterapia intensiva (R DA EPOCH), obteniendo una buena respuesta terapéutica hasta la fecha. Como no hay comunicaciones de las que tengamos conocimiento, de PTLD y "triple expresor" tratado con este esquema, creemos que esta información puede ser de utilidad ante la aparición de casos similares.

\section{Referencias}

1. Dierickx D, Haberman TM. Post-transplantation lymphoproliferative disorders in adults. N Engl J Med 2018; 378: 549-62.

2. Siegel RL, Miller KD, Jemal A. Cancer statistics, 2016. CA Cancer J Clin 2016; 66: 7-30.

3. de Fijter JW. Cancer and mTOR inhibitors in transplant recipients. Transplantation 2017; 10145-55.

4. Opelz G, Döhler B. Lymhomas after solid organ transplantation: a collaborative transplant study report. Am J Transplant 2004; 4: 222-30.

5. Clarke CA, Morton LM, Lynch C, Pfeiffer RM, Hall EC, Gibson TM, et al. Risk of lymphoma subtypes after solid organ transplantation in the United States. British Journal of Cancer 2013; 109: 280-8.

6. Kinch A, Baecklund E, Backlin C, Ekman T, Molin D, Tufveson G, et al. A population-based study of 135 lymphomas after solid organ transplantation: the role of Epstein-Barr virus, hepatitis $\mathrm{C}$ and diffuse large-B-cell 
lymphoma subtype in clinical presentation and survival. Acta Oncol 2014; 53: 669-79.

7. Luskin MR, Heil DS, Tan KS, Choi S, Stadtmauer EA, Schuster SJ, et al. The impact of EBV status on characteristics and outcomes of posttransplantation lymphoproliferative disorder. Am J Transplant 2015; 15: 2665-73.

8. The international Non-Hodgkin's Lymphoma Prognostic Factor Proyect. predictive model for aggressive non-Hodgkin's lymphoma. N Engl J Med; 329: 987.

9. Wang W, Hu S, Lu X, Young KH, Medeiros LJ. Triple-hit B-cell lymphoma with MYC, BCL2, and BCL6 translocations/rearrangements: clinicopathologic features of 11 cases. Am J Surg Pathol 2015; 39: 1132-9.

10. Swerdlow S, Campo E, Harris NL, Jaffe ES, Pileri SA, Stein $\mathrm{H}$, et al. WHO Classification of Tumours of Haematopoietic and Lymphoid Tissues. International Agency for Research on Cancer Lyon, 2017.

11. Rosenthal A, Younes A. High grade B-cell lymphoma with rearrangements of MYC and BCL2 and/or BCL6: Double hit and triple hit lymphomas and double expressing lymphoma. Blood Reviews 2016; 1-6.

12. Akyurek N, Uner A, Benekli M, Barista I. Prognostic significance of MYC, BCL2, and BCL6 rearrangements in patients with diffuse large B-cell lymphoma treated with cyclophosphamide, doxorubicin, vincristine, and prednisone plus rituximab. Cancer 2012; 118: 4173-83.

13. Johnson NA, Savage KJ, Ludkovski O, Ben-Neriah S,
Woods R, Steidl C, et al. Lymphomas with concurrent BCL2 and MYC traslocations; the critical factors associated with survival. Blood 2009; 114 (11): 2273-9.

14. Peña C, Marti M, Villegas $P$, Undurraga MS. Hipercalcemia, peak monoclonal y leucemización como presentación de un linfoma de células B de alto grado, con reordenamiento de MYC, BCL-2 y BCL-6 (Triple Hit). Caso clínico. Rev Med Chile 2017; 145: 1485-9.

15. Herrera AF, Mei M, Low L, Kim HT, Griffin GK, Song JY, et al. Relapsed or refactory double-expresor and double-hit lymphomas have inferior progression-free survival after autologous stem cell transplantation. J Clin Oncl 2017, 35: 24-31.

16. Reagan PM, Davies A. Current treatment of double hit and double expressor lymphoma. Hematology Am Soc Hematol Educ Program 2017; 295-7.

17. Wilson WH, sin-Ho J, Pitcher BN. Phase III randomized study of R-CHOP versus R-DA-EPOCH and molecular analysis of untreated diffuse large b cell lymphoma. Blood 2016; 128: 496.

18. Dierickx D, Tousseyn T, Gheysens O. How I treat posttransplant lymphoproliferative disorders. Blood 2015; 126: 2274-83.

19.- DeStefano C, Malkovska V, Rafei H, Shenoy A, Fitzpatrick K, Aggarwal A, et al. DA-EPOCH-R for Post-Transplant Lymphoproliferative Disorders (PTLD). doi: 10.1111/ejh.12904 (Ahead of print). 